\title{
Design of a Long-Pass Filter with Effects on Fluorescence Image Observation for Surgical Fluorescence Microscope Applications
}

\author{
Jinkyu Kim ${ }^{1,2,3,4}$, Kicheol Yoon ${ }^{1,2}$ and Kwanggi Kim 1,2,4,5,*(D) \\ 1 Medical Devices R\&D Center, Gachon University Gil Medical Center, 21, 774 beon-gil, Namdong-daero, \\ Namdong-gu, Incheon 21565, Korea; jinkyup98@gmail.com or jinkyu98@ucla.edu (J.K.); \\ kcyoon98@gachon.ac.kr (K.Y.) \\ 2 Department of Biomedical Engineering, College of Medicine, Gachon University, \#701, 38-13, 3 Dokjom-ro, \\ Namdong-gu, Incheon 21565, Korea \\ 3 Department of Chemistry and Biochemistry, College of Life Science, University of California, \\ Los Angeles (UCLA), 405 Hilgard Ave., Los Angeles, CA 90095, USA \\ 4 Department of Biomedical Engineering, College of Health Science, Gachon University, 191 Hambangmoe-ro, \\ Namdong-gu, Incheon 21936, Korea \\ 5 Department of Health Sciences and Technology, Gachon Advanced Institute for Health Sciences and \\ Technology (GAIHST), Gachon University, 38-13, 3 Dokjom-ro, Namdong-gu, Incheon 21565, Korea \\ * Correspondence: kimkg@gachon.ac.kr; Tel.: +82-32-458-2881
}

Citation: Kim, J.; Yoon, K.; Kim, K. Design of a Long-Pass Filter with

Effects on Fluorescence Image

Observation for Surgical Fluorescence Microscope Applications. Symmetry 2021, 13, 1571. https://doi.org/ $10.3390 /$ sym 13091571

Academic Editors: Jan Awrejcewicz and Dumitru Baleanu

Received: 19 July 2021

Accepted: 25 August 2021

Published: 26 August 2021

Publisher's Note: MDPI stays neutral with regard to jurisdictional claims in published maps and institutional affiliations.

Copyright: (C) 2021 by the authors Licensee MDPI, Basel, Switzerland. This article is an open access article distributed under the terms and conditions of the Creative Commons Attribution (CC BY) license (https:/ / creativecommons.org/licenses/by/ $4.0 /)$.
Abstract: The goal of oncological surgery is to completely remove the tumor. Tumors are often difficult to observe with the naked eye because of the presence of numerous blood vessels and the fact the colors of the tumor and blood vessels are similar. Therefore, a fluorescent contrast medium using a surgical microscope is used to observe the removal status of the tumor. To observe the tumor removal status using a fluorescent contrast agent, fluorescence is expressed in the tumor by irradiating with an external light source, and the expressed tumor can be confirmed through a surgical microscope. However, not only fluorescence-expressed tumors are observed under a surgical microscope, but images from an external light source are also mixed and observed. Therefore, since the surgical microscope is connected to a filter, the quality of the diagnostic image is not uniform, and it is difficult to achieve a clear observation. As a result, an asymmetric image quality phenomenon occurs in the diagnostic images. In this paper, a filter with high clarity that provides a symmetrical observation of diagnostic images is developed and manufactured.

Keywords: surgical microscope; filter; diagnostic image; asymmetric image quality phenomenon; symmetrical observation

\section{Introduction}

The purpose of surgery is to completely remove malignant tumors with high dislocation rate and strong invasiveness [1]. Numerous blood vessels are distributed inside the tumor. Because the color of the tumor and blood vessels are similar, it is often difficult to visually observe the tumor removal status. Therefore, a yellow-dye $(530-560 \mathrm{~nm}$ ) fluorescence contrast medium is injected into the body to distinguish blood vessels from tumors, and the surgical area is exposed to an external light source $(405 \mathrm{~nm})$ to induce fluorescence emission from the tumor [2].

The wavelength and color images that fluoresce in the tumor can be observed on an external monitor through the camera of a surgical microscope [3]. However, since the surgical microscope captures the fluorescence color image of the tumor and the color image of the external light source together through the camera, the external monitor observes the color image of the external light source and the fluorescence color image of the tumor mixed. Therefore, it is impossible to accurately observe the tumor on the monitor. To overcome this problem, a long-pass filter is applied to the surgical microscope. Therefore, the long-pass filter passes the fluorescence wavelength for the tumor and blocks the wavelength for 
the external light source, so that only the fluorescence-expressing tumor is shown when observed on an external monitor. An off-the-shelf product must be used to mount the filter on a surgical microscope. As for the filter, it is important to have a sharp wavelength blocking, symmetry pass rate and cut rate. However, it is not easy to produce a ready-made filter suitable for a surgical microscope. The long-pass filter (FELH 0500) provided by Thorlabs (city, state abbrev if USA, country) has a transmission efficiency and blocking rate of $80 \%$ and $0.01 \%$ at $500 \mathrm{~nm}$, respectively. At this time, since the blocking efficiency of the long-pass filter is $0.01 \%$ but the transmission efficiency is $80 \%$, the image quality for diagnosing a tumor in the fluorescent state is reduced by $20 \%$, thereby preventing accurate diagnosis. Therefore, the filter has $100 \%$ asymmetry in pass and cut efficiency. For this reason, studies are being conducted to design a filter suitable for a surgical microscope. Among them, the optical filter using CMOS has an efficiency of 50\% [4]. Filters using $\mathrm{S}_{\mathrm{i}} \mathrm{O}_{2}$ and $\mathrm{T}_{\mathrm{i}} \mathrm{O}_{2}$ have an efficiency of $80 \%$ at $510-550 \mathrm{~nm}$ [5]. A filter using silicon photomultipliers has a transmission efficiency of $95 \%$ [6]. The transmission efficiency of the optical filter using silicon is $90 \%$ [7]. As a result of analyzing the efficiency, the transmission efficiency of the filter for application to a surgical microscope must have a symmetry between $98-100 \%$. The reason is that when the transmission (T) efficiency is $98 \%$ or more, the reflection (OD) efficiency approaches $0 \%$. Therefore, when the pass efficiency of the filter is $100 \%$ in theory, the reflection efficiency is $0 \%$, so it has symmetry.

In addition, if the transmittance and reflectance are asymmetric, the surgical diagnosis image is blurred in the surgical microscope, and if the reflectance is low the wavelength irradiated from the light source and the fluorescence wavelength are mixed and the image is broken. Therefore, applying a long-pass filter having high transmission efficiency and low reflection efficiency can provide symmetrical image efficiency. For this, the transmission efficiency and reflection efficiency of the long-pass filter must be $98 \%$ or more and within $0.2 \%$. In this paper, we propose a symmetric long-pass filter design with high transmittance that can pass only fluorescence wavelengths.

\section{Materials and Design Methods}

As shown in Figure 1, an optical filter, which is a micro-disk type, is an asymmetrical multilayered structure, each with different materials. Such materials include $\mathrm{S}_{\mathrm{i}} \mathrm{O}_{2}$ and $\mathrm{T}_{\mathrm{i} 3} \mathrm{O}_{5}$, which are deposited on top of a glass substrate.

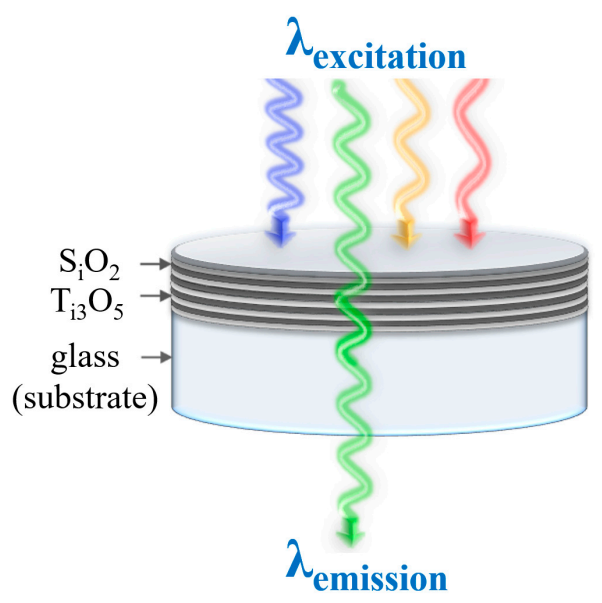

Figure 1. An excitation light wavelength passing through a multilayered optical filter.

An optical filter can block an undesired region of wavelength. However, when light passes through the filter, the light intensity is distributed asymmetrically due to energy (power) loss [8]. This power loss (PL) is caused by the parasitic resistance of each material comprising the filter and can be calculated by using Equation (1). Equation (2) shows that parasitic resistance $(R)$ is directly proportional to the specific resistance $(\rho)$ of the material. 
The parasitic values for glass, $\mathrm{S}_{\mathrm{i}} \mathrm{O}_{2}$, and $\mathrm{T}_{\mathrm{i} 3} \mathrm{O}_{5}$ are $10 \times 10^{11}$ (to $10^{15}$ ) $\Omega \cdot \mathrm{m}, 2.3 \times 10^{3} \Omega \cdot \mathrm{m}$, and $4.7 \times 10^{-3} \Omega \cdot \mathrm{m}[9-12]$ :

$$
I_{m}=\left(\frac{1-R}{1+R}\right)^{2}
$$

where $l_{m}$ represents the amount of energy lost due to the parasitic resistance:

$$
R=\rho \frac{l}{A}
$$

where $\rho, l$, and $A$ are specific resistance, length, and dimension of the material, respectively.

The essential parameters of a filter are wavelength, transmittance $(\mathrm{T})$, and optical density (OD), and the latter two can be measured by using Equations (3) and (4). Theoretically speaking, a filter should have T of $100 \%$ and OD and PL of 0 :

$$
\begin{gathered}
T=10^{-O D} \times 100[\%] \\
O D=-\log \left(\frac{I_{o}}{I_{p}}\right)
\end{gathered}
$$

where $I_{o}$ and $I_{p}$ are the intensity of incident light and transmitted light, respectively. However, as shown in Figure 2, when light passes through a layer, it loses additional energy $\left(l_{T}\right)$.

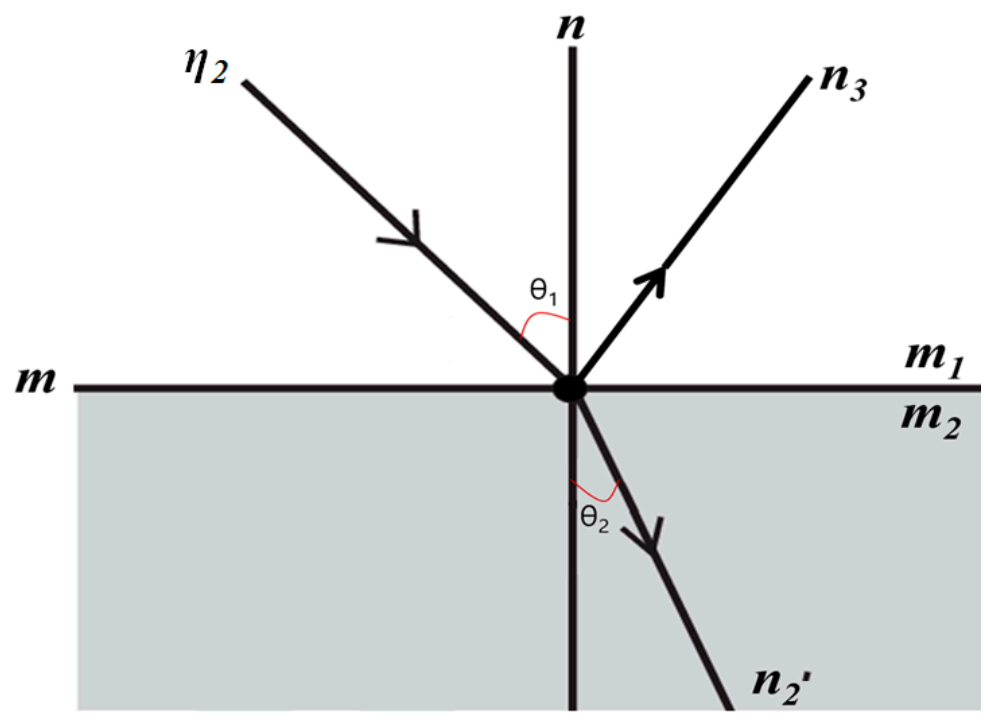

Figure 2. Characteristics of light according to the Snell's law $\left(m_{1}\right.$ and $m_{2}$ : material of 1 layer and 2 layer, $\eta_{2}$ : incident light, $\eta_{2}^{\prime}$ : absorption light, and $\eta_{3}$ : reflection light).

Equation (5) shows the way to calculate the extent of energy loss. It is caused by asymmetrical changes in refractive index $(\eta)$, light reflection $(\Gamma)$, and light absorption $(\alpha)$ of any part. They can be calculated by using Equations (6)-(8). Epically, the Equation (6) represents the Snell's law, which can be used to calculate changes in $\eta$.

$$
\begin{gathered}
I_{t}=\left(\frac{1-\eta}{1+\eta}\right)^{2} \\
\eta_{2} \sin \theta_{1}=\eta_{2}^{\prime} \sin \theta_{2} \\
\Gamma=\frac{\left(\eta_{2}^{\prime} \cos \theta_{1}-\eta_{2} \cos \theta_{2}\right)}{\left(\eta_{2}^{\prime} \cos \theta_{1}+\eta_{2} \cos \theta_{2}\right)} \\
\alpha=\frac{4 \pi k}{\lambda}
\end{gathered}
$$


where $k$ is the extinction coefficient, which is $1 \times 10^{-5}$, and $\lambda$ is a wavelength of light.

Consequently, the efficiency of a filter $\left(n_{e f}\right)$ decreases as a result of asymmetrical multiple PL and can be calculated by using Equation (9). Equation (10) shows a way to calculate the overall PL:

$$
n_{\text {ef }}=\left(\frac{P_{\text {out }}}{P_{\text {in }}}\right) \times 100
$$

where $P_{\text {in }}$ and $P_{\text {out }}$ are input power and output power, respectively.

$$
P L=-10 \log O D
$$

As shown in Figure 3a, the long-pass filter fabricated for this study is made up of a glass substrate, $\mathrm{S}_{\mathrm{i}} \mathrm{O}_{2}, \mathrm{~T}_{\mathrm{i} 3} \mathrm{O}_{5}, \mathrm{~L}_{\mathrm{iF}}$, and $\mathrm{I}_{\mathrm{T}} \mathrm{O}$. Each material is deposited on top of each other through physical vapor deposition $\left(\mathrm{P}_{\mathrm{VD}}\right)$. The $\eta$ of $\mathrm{S}_{\mathrm{i}} \mathrm{O}_{2}$ and $\mathrm{T}_{\mathrm{i} 3} \mathrm{O}_{5}$ are 2.3 and 1.5, respectively. In addition, $m, l, p, t$, and $h$ represent the height of each layer, with each letter corresponding to $1.2,4,1,2 \mu \mathrm{m}$, and $0.2 \mathrm{~mm}$, respectively.

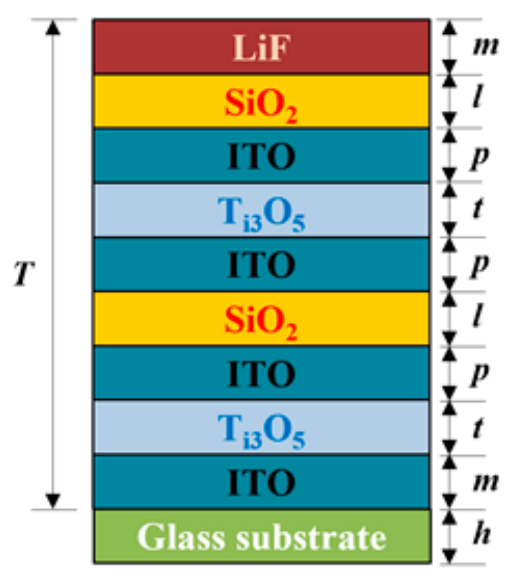

(a)

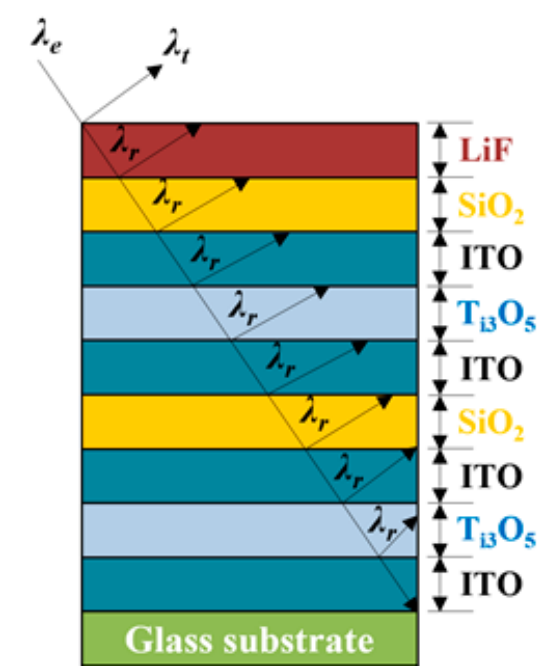

(b)

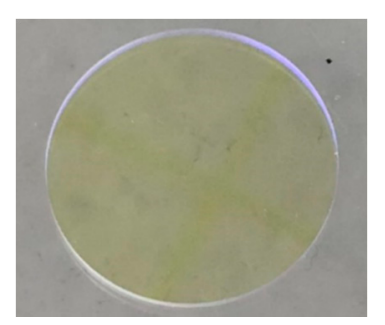

(c)

Figure 3. Structure of a fabricated filter (a) layer (b) light propagation of a layer (c) fabricated filter.

Figure $3 \mathrm{~b}$ shows the passage of light when it passes through this filter. In this figure, $\lambda_{r}$ and $\lambda_{t}$ are the reflected and transmitted light, respectively. By adjusting the heights of each layer excluding the glass substrate, the refraction, reflection, and transmission of light can be controlled. Finally, Figure $3 c$ displays the image of the fabricated filter.

\section{Experimental Results}

The purpose of this experiment is to find out the effects of a long-pass filter on the quality (symmetrical distribution of light) of a fluorescence image. For fluorescence image analysis, $0.0102 \mathrm{mM}$ of FL solution was prepared in a vial by adding $0.5 \mathrm{~mL}$ of saline and $0.02 \mathrm{~mL}$ of FL (FLUORESCITE ${ }^{\circledR}$ Injection 10\%/Alcon Korea, Korea Alcon. Co. Ltd. Seoul, Republic of Korea). For fluorescence excitation, a $405 \mathrm{~nm}$ light emitting diode (LED, M405L2-C1/Thorlabs) was used. Its irradiation wavelength, forward voltage, and bandwidth are $405 \mathrm{~nm}, 3.8 \mathrm{~V}$, and $13 \mathrm{~nm}$, respectively. The power intensity emitted from this LED was $10 \mathrm{~mW}$, which was measured by using a power meter (PM100D/Thorlabs). The distance from the light source to the sample was fixed to $5 \mathrm{~cm}$, and the angle of incidence was $45^{\circ}$. The beam angle was measured to be $2.44^{\circ}$. For fluorescence observation, a near-infrared (NIR) camera (Lt-225c, Teledyne Lumenera. Co. Ltd. Ottawa, ON, Canada) was used. This camera has a pixel size of $5.5 \mu \mathrm{m}$, resolution $2048 \times 1088$ pixels, and a frame rate of $170 \mathrm{fps}$. 
Based on this setup, three different fluorescence images were recorded. The first image was taken without a filter. Another image was taken with a conventional long-pass filter (FELH0500/Thorlabs) that has a diameter of $25 \mathrm{~mm}$ and a thickness of $3.5 \mathrm{~mm}$. The other image was taken with a fabricated long-pass filter that has a diameter of $25 \mathrm{~mm}$ and a thickness of $1.0 \mathrm{~mm}$.

Lastly, the intensity of fluorescence light that passed through a filter was measured by using a spectrometer (FLAME-S-VIS-NIR-ES/Ocean Insight, Orlando, FL, USA). The filter was positioned between the sample and the spectrometer.

Fluorescent contrast medium $(0.8 \mathrm{~mL})$ diluted with fluorescent dye and normal saline was injected into a $1 \mathrm{~mL}$ vial as shown in Figure 4 . There were three vials into which the fluorescent contrast agent was injected, and the vials were divided into unfiltered vials and filter-applied vials. The applied filters were a conventional filter and a newly designed filter, and all three vials were irradiated with an external light source of $405 \mathrm{~nm}$.
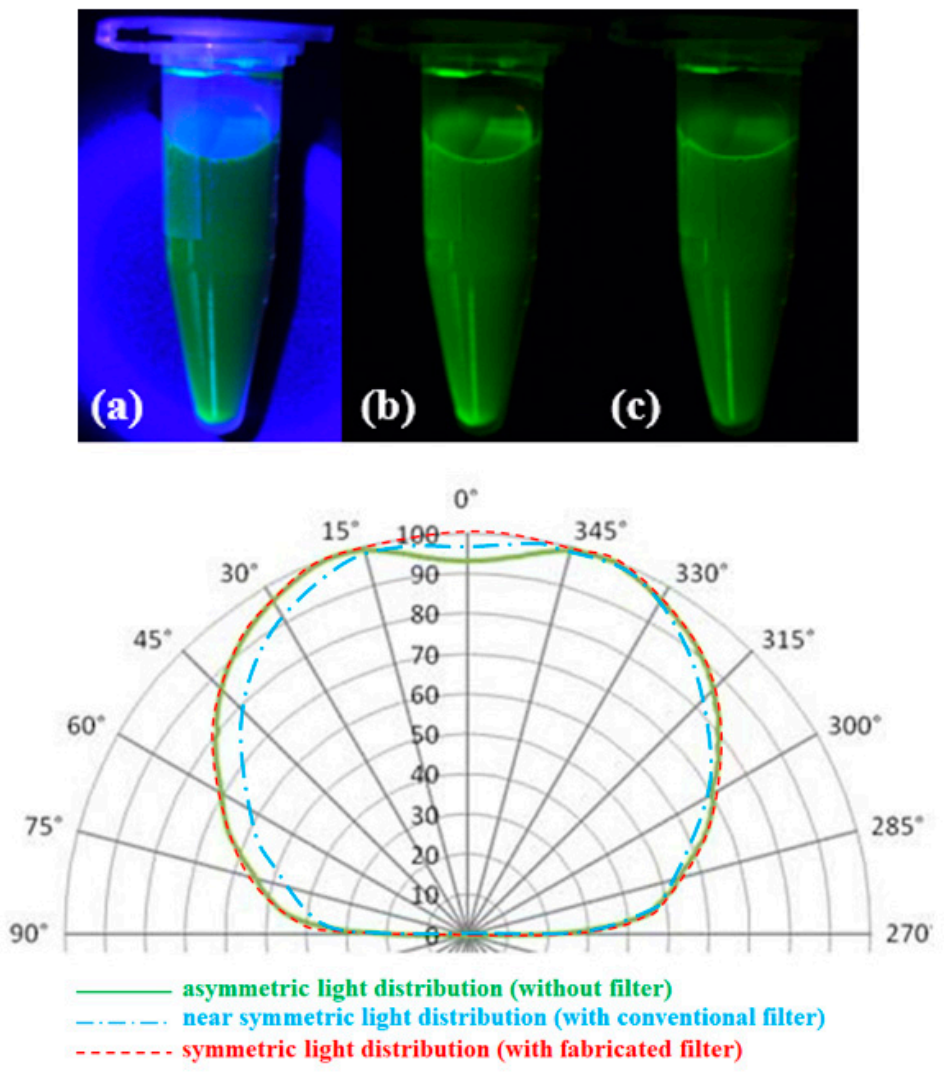

(d)

Figure 4. Fluorescence images taken (a) without a filter, (b) with a conventional filter, (c) with a fabricated filter, and (d) fluorescence polar chart of symmetrical light distribution.

In the first vial (see Figure 4a) irradiated with an external light source of $405 \mathrm{~nm}$, the fluorescence color was blurred, and the color was changed. Therefore, the light did not match and changed to an asymmetric intensity. When diagnosing an actual lesion, it will be difficult to distinguish and observe tumors and blood vessels with similar colors. In the second vial (conventional filter) (see Figure $4 \mathrm{~b}$ ) irradiated with an external light source of $405 \mathrm{~nm}$, the fluorescence color was accurately displayed. However, the fluorescent color is slightly dimmed, the light is not clear as a whole, and the light intensity is not symmetrical as a whole. That is, since the left side is bright and the right side is dark, the brightness of the light is asymmetric. In the third vial (designed a filter) (see Figure 4c) irradiated with an external light source of $405 \mathrm{~nm}$, the fluorescence color was accurately displayed, and 
the color was also clearly displayed. In addition, the light intensity was symmetrically and uniformly distributed.

The emission wavelength compared to the excitation wavelength was measured when an external light source $(405 \mathrm{~nm})$ was irradiated on a vial without a filter and a vial with a filter (conventional filter and designed a filter). At this time, separate observations were made for the peak wavelength when no filter was applied and when the filter was applied.

In Figure $4 \mathrm{a}$, the left side of the vial is blurry, and the right side has dark shadows. In addition, a blue background is observed outside the vial because the wavelength of ambient light sources at $405 \mathrm{~nm}$ was unblocked. Therefore, at $15^{\circ}-345^{\circ}$ of Figure $4 \mathrm{~d}$, slight bending was observed, making the image asymmetrical. Figure $4 \mathrm{~b}$ is more vivid than Figure $4 \mathrm{a}$, but the right side of the vial is darker than the left side. Although the $405 \mathrm{~nm}$ wavelength was blocked by the filter, materials used in this filter failed to reduce transmission loss, and therefore a dark image was produced. In Figure 4c, the image looks clear and symmetric. That is, the filter used to observe this image successfully reflected ambient light sources. In addition, this filter reduced transmission loss, producing a clear fluorescence image. At $15^{\circ}-345^{\circ}$ in Figure $4 \mathrm{~d}$, flatness was formed, and no bending occurred.

Figure 5 shows fluorescence emission from the sample. The peak intensity values, along with the corresponding wavelengths, are recorded in Table 1. The average peak intensity was obtained at wavelengths of $531.89 \mathrm{~nm}( \pm 1.19 \mathrm{~nm})$. The peak intensity achieved by the fabricated filter was $50,483.45$ in counts, whereas that achieved by the conventional filter was $46,103.17$ in counts. Considering that the quality of an image increases along with an increase in peak intensity, it is determined that the image quality of Figure $4 \mathrm{c}$ is higher than that of Figure $4 \mathrm{~b}$ [13].

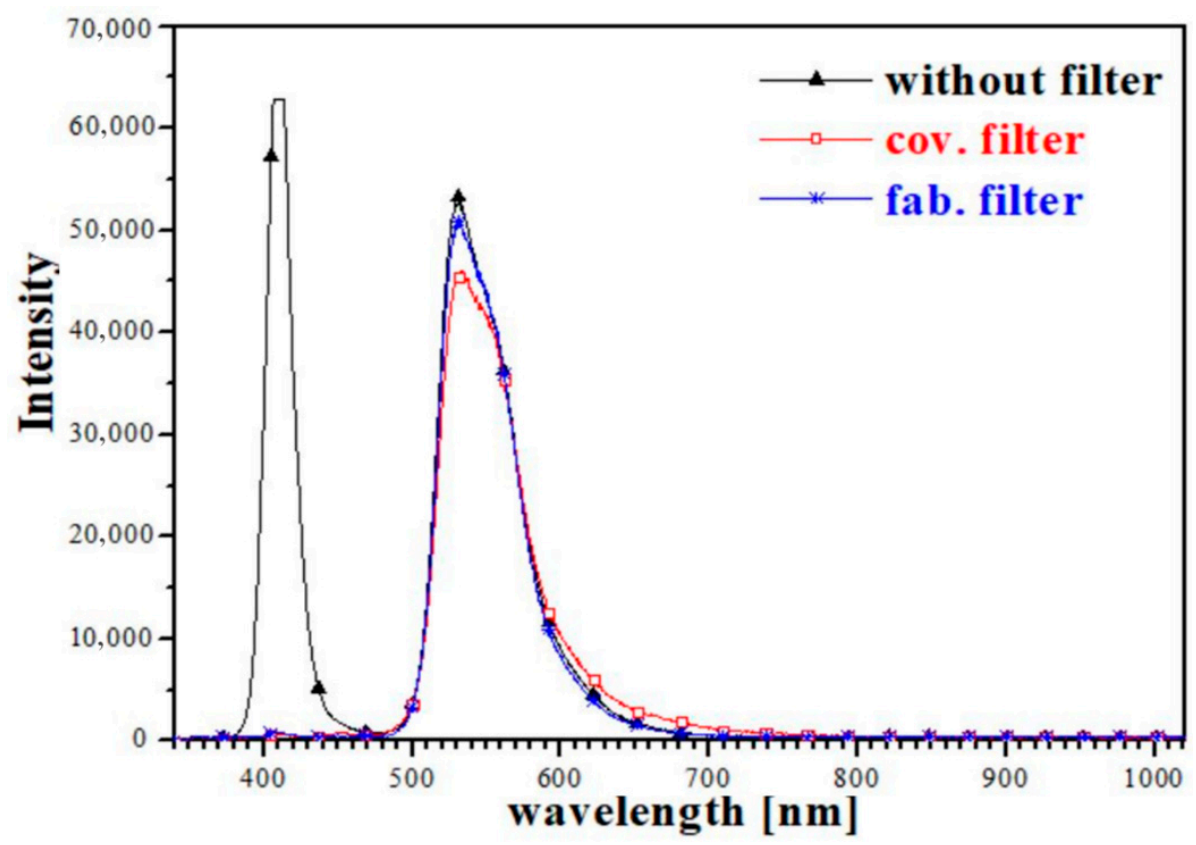

Figure 5. Spectroscopy graph of fluorescence emission (cov: conventional filter, fab.: fabricated filter).

Table 1. Fluorescence intensity and wavelength for each trial, and the three parameters of a filter.

\begin{tabular}{cccc}
\hline & Without Filter & $\begin{array}{c}\text { Conventional Filter } \\
\text { [Long-Pass Filter, FEL0500] }\end{array}$ & Fabricated Filter \\
\hline Intensity [counts] & $52,775.74$ & $46,103.17$ & $50,483.45$ \\
Wavelength [nm] & 532.49 & 532.49 & 530.7 \\
Power loss [dB] & N/A & -0.879251 & -0.192853 \\
OD [\%] & N/A & 0.0879251 & 0.0192853 \\
T [\%] & N/A & 87.3567 & 95.6565 \\
\hline
\end{tabular}


Then, by plugging the peak intensity values into Equations (3), (4) and (10), PL, OD, and $\mathrm{T}$, which are the essential parameters of a filter, were calculated and displayed in Table 1.

Referring to the table, it was possible to confirm that the PL and OD were lower, and the $\mathrm{T}$ value was higher in the filter manufactured to obtain a fluorescence image with uniform light intensity and symmetrical high clarity compared to the conventional filter. Since PL, OD, and T have a correlation with symmetry, it was found that the lower the PL of the filter, the clearer the fluorescence image and the symmetrical change in light intensity.

\section{Discussion}

The proposed filter is made up of a glass substrate, $\mathrm{S}_{\mathrm{i}} \mathrm{O}_{2}, \mathrm{~T}_{\mathrm{i} 3} \mathrm{O}_{5}$, Tif, and ITO. The materials used in the proposed filter are inexpensive and are readily available on the market. By adjusting the thickness of each material, the filter's performance can be significantly improved. Although there exist many different types of filters on the market, their wavelength bands are not suitable for use in diagnostic devices. In addition, such filters are not designed to reduce the loss of transmitted light. As a result, the blurry images are produced, making it difficult to observe the lesion. Therefore, we decided to make our own filter. The main advantages this filter has are low unit price and excellent image processing.

In Figure 3, T (total thickness) must be within 1/4 wavelength. The types of materials and the thickness of the filter are related to the refractive index (incident, reflection, refraction, interference, etc.) [10]. For example, the refractive indices of $\mathrm{S}_{\mathrm{i}} \mathrm{O}_{2}$ and $\mathrm{T}_{\mathrm{i} 3} \mathrm{O}_{5}$ are 2.3 and 1.5, which are optimal for the filter. These refractive indices are achieved by adjusting the thickness ratio of $\mathrm{S}_{\mathrm{i}} \mathrm{O}_{2}$ and $\mathrm{T}_{\mathrm{i} 3} \mathrm{O}_{5}$ to 2:1. In this study, the thickness of $\mathrm{S}_{\mathrm{i}} \mathrm{O}_{2}$ and $\mathrm{T}_{\mathrm{i} 3} \mathrm{O}_{5}$ were $4 \mu \mathrm{m}$ and $2 \mu \mathrm{m}$, respectively. Similarly, the thickness of ITO was fixed at $1.2 \mu \mathrm{m}$ to reduce interference, and that of absorber (Lif) was fixed at $1 \mathrm{um}$ to increase the incident light. These characteristics allow the total $(\mathrm{T})$ thickness to be $1 / 4$ of the wavelength (by definition, a 1:1 ratio).

The refractive index and reflectance of the filter depend on the properties of materials $\left(\mathrm{S}_{\mathrm{i}} \mathrm{O}_{2}\right.$ and $\left.\mathrm{T}_{\mathrm{i} 3} \mathrm{O}_{5}\right)$, such as their thickness. Therefore, the types and thickness of materials are carefully selected and controlled to minimize the refraction and reflection. As mentioned above, it can be achieved by adjusting the entire thickness of the filter to be $1 / 4$ of wavelength. Therefore, the filter should be carefully designed to make the entire filter $(\mathrm{T})$ in a $1: 1$ ratio.

Fluorescence-guided surgery or diagnosis is widely performed in real life. In this technique, a surgical fluorescence microscope must produce a high-quality image for better diagnosis or treatment. Throughout this study, a long-pass filter proved to be the major contributor to the production of a clear fluorescence symmetrical image as it blocks unwanted light. In addition, the results of this study indicate that for better fluorescence observation, a filter with low OD and PL and high T need to be used. In fact, it is not an exaggeration to argue that a surgical camera whose performance is phenomenal is of no use without an appropriate filter. Thus, it can be assumed that the case studies mentioned above used inappropriate filters, resulting in the production of obscure fluorescence images [14-17]. For research studies to produce high-quality fluorescence images, researchers need to either use a filter with low PL or fabricate a filter with materials that can reduce PL.

One possible reason surgeons having never raised an issue regarding unclear fluorescence images is that they are simply used to performing surgery with such images. However, this is a serious problem because, with obscure images, surgeons will not be able to easily distinguish tumors from blood vessels. In addition, looking at unclear images for a long period of time will cause eye strain on them, which may lead to a disturbance in their concentration. Hence, introducing a filter with low PL will likely increase the success rate of tumor resection.

FL fluoresces even with a small droplet of saline. Since the main purpose of this study is to investigate the effects of a long-pass filter on a fluorescence image, this paper is premised on the assumption that the peak fluorescence emission intensity is reached at the 
concentration of $0.0102 \mathrm{mM}$. In a clinical trial, however, the concentration of FL solution should be adjusted according to the type of subject being tested on. Additionally, a further clinical trial needs to be performed on living subjects to validate that PL, OD, and T of a filter have effects on the quality of a fluorescence image.

As a result of analyzing the application fields, the analysis shows that the proposed filter is applicable to not just tumor diagnosis but also in other fields. Reference [18] describes tumor-associated macrophages (M1/M2), whose fluorescence excitation wavelength and emission wavelength are $488 \mathrm{~nm}$ and 509-620 nm (peak emission $560 \mathrm{~nm}$ ), respectively. These data show that the proposed filter corresponds to the wavelength band of [18] and therefore can be used for [19].

In addition, the emission wavelength of fibroblasts [20] is 520-560 nm [21]. In the fluorescent wavelength of pericytes [22], the excitation wavelength is $405 \mathrm{~nm}$ and the emission wavelengths are $488 \mathrm{~nm}, 543 \mathrm{~nm}$, and $633 \mathrm{~nm}$, respectively [23]. Therefore, the proposed filter is applicable to tumor observation of macrophages, fibroblasts, and pericytes.

In general, tumors are located near blood vessels [24] but it is difficult to observe tumors with naked eye because of similarity of colors of the tumor and blood vessels. Therefore, for better diagnosis, a fluorescent contrast agent is administered to patients to highlight blood vessels, allowing for clear distinction between the two areas. The resulting fluorescence is then viewed under the surgical fluorescence microscope [24]. Considering that this fluorescence-based approach contributes to better diagnosis of diseases, we suggest providing training programs for medical students and staffs.

In another application analysis, it is expected that the proposed filter can be used not only for fluorescence-guided tumor diagnosis but also in forensic fields (fingerprint: 505-610 nm) [25,26].

\section{Conclusions}

In this paper, a high-efficiency long-pass filter was designed, and the designed filter was used to observe the image and measure the waveform of the fluorescence contrast medium.

In this study, the effect of long-pass filter on fluorescence image observation was investigated because the current surgical fluorescence microscope cannot produce clear images due to asymmetric light distribution. In the study, fluorescence images were taken both in the absence and the presence of a long-pass filter. By comparing the fluorescence images taken with and without a filter, it was revealed that a filter is a necessary component as it blocks most of the undesired light.

To discover characteristic sets a filter should possess for clear fluorescence image production, this study introduced a fabricated long-pass filter and compared its performance with a conventional filter's performance. By measuring the fluorescence emission intensity after passing through each filter, OD, PL, and T of each filter were calculated. The results indicated that a filter with lower OD and PL and higher T transmitted a greater amount of light. Given that the more light transmitted through, the sharper the image, we conclude that filters with low OD and PL and high T produce sharp images due to the symmetrical distribution of light. By using a filter with low OD and PL and high T, surgeons will be able to acquire a clear fluorescence image and thus better perform tumor-resection. Furthermore, such filters will allow researchers to better interpret their results in their research studies.

Author Contributions: J.K. analysed the theory and performed experiments, and K.Y. worked on the mathematical analysis. K.K. advised the other researchers. All authors have read and agreed to the published version of the manuscript.

Funding: This research was supported by the Ministry of Science and ICT (MSIT), Korea, under the Information Technology Research Center (ITRC) support program (IITP-2020-20170-01630) supervised by the Institute for Information \& Communications Technology Promotion (IITP), and G-ABC (FRD2019-11-02(3). 
Institutional Review Board Statement: Not applicable.

Informed Consent Statement: Not applicable.

Data Availability Statement: The data presented in this study are available on request from the corresponding authors. The data are not publicly available due to privacy restrictions.

Acknowledgments: Jinkyu Kim and Ki-Cheol Yoon contributed equally to this effort and must be considered lead (first) co-authors.

Conflicts of Interest: The authors declare no conflict of interest.

\section{References}

1. $\quad$ Park, J.W.; Sohn, D.K.; Hong, C.W.; Han, K.S.; Choi, D.H.; Chang, H.J.; Lim, S.-B.; Choi, H.S.; Jeong, S.-Y. The usefulness of preoperative colonoscopic tattooing using a saline test injection method with prepackaged sterile India ink for localization in laparoscopic colorectal surgery. Surg. Endosc. 2007, 22, 501-505. [CrossRef]

2. Arteaga-González, I.; Martín-Malagón, A.; Fernández, E.M.L.-T.; Arranz-Durán, J.; Parra-Blanco, A.; Nicolas-Perez, D.; QuinteroCarrión, E.; Luis, H.D.; Carrillo-Pallares, A. The Use of Preoperative Endoscopic Tattooing in Laparoscopic Colorectal Cancer Surgery for Endoscopically Advanced Tumors: A Prospective Comparative Clinical Study. World J. Surg. 2006, 30, 605-611. [CrossRef]

3. Reinhold, B. Latest results of 5-ALA-based fluorescence diagnosis and other medical disciplines. Proc. SPIE 1999, 3563, 90-99.

4. Yokogawa, S.; Burgos, S.P.; Atwater, H.A. Plasmonic Color Filters for CMOS Image Sensor Applications. Nano Lett. 2012, 12, 4349-4354. [CrossRef]

5. Shin, K.-S.; Kim, Y.-H.; Min, J.-A.; Kwak, S.-M.; Kim, S.K.; Yang, E.G.; Park, J.-H.; Ju, B.-K.; Kim, T.-S.; Kang, J.Y. Miniaturized fluorescence detection chip for capillary electrophoresis immunoassay of agricultural herbicide atrazine. Anal. Chim. Acta 2006, 573-574, 164-171. [CrossRef] [PubMed]

6. Mazzillo, M.C.; Maddiona, L.; Rundo, F.; Sciuto, A.; Libertino, S.; Lombardo, S.; Fallica, G. Characterization of SiPMs With NIR Long-Pass Interferential and Plastic Filters. IEEE Photonics J. 2018, 10, 1-12. [CrossRef]

7. Hinken, D.; Schinke, C.; Herlufsen, S.; Schmidt, A.; Bothe, K.; Brendel, R. Experimental setup for camera-based measurements of electrically and optically stimulated luminescence of silicon solar cells and wafers. Rev. Sci. Instrum. 2011, 82, 33706. [CrossRef] [PubMed]

8. Saleh, B.; Teich, M.; Slusher, R.E. Fundamentals of Photonics. Phys. Today 1992, 45, 87-88. [CrossRef]

9. Serway, R.A. Principles of Physics, 2nd ed.; Saunders College Publishing: Fort Worth, TX, USA, 1998; Volume 155, ISBN 978-0-03020457-9.

10. Chen, Y.; Mao, J. Sol-gel preparation and characterization of black titanium oxides $\mathrm{Ti}_{2} \mathrm{O}_{3}$ and $\mathrm{Ti}_{3} \mathrm{O}_{5}$. J. Mater. Sci. Mater. Electron. 2014, 25, 1284-1288. [CrossRef]

11. Copper and Electricity. In Resistance and Resistivity; The Association for Science Education: Hatfield, UK, 2014. Available online: http:/ / resources.schoolscience.co.uk/CDA/16plus/ copelech2pg1.html (accessed on 16 June 2020).

12. Park, S.S.; Kwon, Y.J.; Chang, H. Design and analysis of optical filters using transmission line theory. J. KICS 1996, 19, 73-76.

13. Kim, S.Y.; Kang, S.S.; Park, J.K.; Cha, B.Y.; Choe, C.W.; Lee, H.W.; Nam, S.H. Fabrication of $\mathrm{Gd}_{2} \mathrm{O}_{3}$ :Eu ${ }^{3+}$ nano phosphor and optical characteristics for high resolution radiation imaging. J. Biomed. Eng. Res. 2007, 28, 148-152.

14. Kuroiwa, T.; Kajimoto, Y.; Ohta, T. Comparison between operative findings on malignant glioma by a fluorescein surgical microscopy and histological findings. Neurol. Res. 1999, 21, 130-134. [CrossRef] [PubMed]

15. Belloch, J.P.; Rovira, V.; Llácer, J.L.; Riesgo, P.A.; Cremades, A. Fluorescence-guided surgery in high grade gliomas using an exoscope system. Acta Neurochir. 2014, 156, 653-660. [CrossRef] [PubMed]

16. Zhang, J.; Al-Nahari, F.; Wang, Z.-F.; Lin, F.-H.; Zhao, Y.-Y.; Xiao, S.-Y.; Liu, J.-M.; Ke, C.; Chen, Z.-H.; Jiang, Y.; et al. Application of fluorescein sodium in the resection of vermis pilocytic astrocytomas. World J. Surg. Oncol. 2017, 15, 46. [CrossRef]

17. He, J.; Yang, L.; Yi, W.; Fan, W.; Wen, Y.; Miao, X.; Xiong, L. Combination of Fluorescence-Guided Surgery with Photodynamic Therapy for the Treatment of Cancer. Mol. Imaging 2017, 16, 1536012117722911. [CrossRef]

18. Li, Y.; Liu, T.-M. Discovering Macrophage Functions Using In Vivo Optical Imaging Techniques. Front. Immunol. 2018,9 , 502. [CrossRef]

19. Zhang, M.; He, Y.; Sun, X.; Li, Q.; Wang, W.; Zhao, A.; Di, W. A high M1/M2 ratio of tumor-associated macrophages is associated with extended survival in ovarian cancer patients. J. Ovarian Res. 2014, 7, 19. [CrossRef]

20. Cangkrama, M.; Wietecha, M.; Mathis, N.; Okumura, R.; Ferrarese, L.; Al-Nuaimi, D.; Antsiferova, M.; Dummer, R.; Innocenti, M.; Werner, $\mathrm{S}$. A paracrine activin A-mDia2 axis promotes squamous carcinogenesis via fibroblast reprogramming. EMBO Mol. Med. 2020, 12, e11466. [CrossRef]

21. Pitas, R.E.; Innerarity, T.L.; Weinstein, J.N.; Mahley, R.W. Acetoacetylated lipoproteins used to distinguish fibroblasts from macrophages in vitro by fluorescence microscopy. Arterioscler. Off. J. Am. Heart Assoc. Inc. 1981, 1, 177-185. [CrossRef]

22. Chen, Z.; Xu, X.H.; Hu, J. Role of pericytes in angiogenesis: Focus on cancer angiogenesis and anti-angiogenic therapy. Neoplasma 2016, 63. [CrossRef] 
23. Seynhaeve, A.L.B.; Oostinga, D.; Van Haperen, R.; Eilken, H.M.; Adams, S.; Adams, R.H.; Hagen, T.L.M.T. Spatiotemporal endothelial cell-pericyte association in tumors as shown by high resolution $4 \mathrm{D}$ intravital imaging. Sci. Rep. 2018, 8, 9596. [CrossRef] [PubMed]

24. Yoon, K.; Kim, K.; Lee, S. A Surgical Pen-Type Probe Design for Real-Time Optical Diagnosis of Tumor Status Using 5Aminolevulinic Acid. Diagnostics 2021, 11, 1014. [CrossRef] [PubMed]

25. Holder, E.H.; Robinson, L.O.; Laub, J.H. The Fingerprint Sourcebook; National Institute of Justice, Office of Justice Programs, U.S. Department of Justice: Washington, DC, USA, 2011.

26. Chuena, L.W.; Eea, K.B. Forensic light sources for detection of biological evidences in crime scene investigation: A review. Malays. J. Forensic Sci. 2010, 1, 17-28. 